\title{
DISTRIBUCIÓN DE INGRESOS Y DETERMINACIÓN DE TARIFAS
}

\section{DISTRIBUTION OF INCOME AND DETERMINATION OF TARIFF}

\author{
Daniel Viera Castillo ${ }^{1}$ \\ Recibido 18 de noviembre de 2005, aceptado 30 de octubre de 2006 \\ Received: November 18, $2005 \quad$ Accepted: October 30, 2006
}

\begin{abstract}
RESUMEN
El presente trabajo pretende demostrar la posibilidad de obtener tarifas de servicios que incorporen la distribución del ingreso y que, simultáneamente, sean eficientes. Se trata de una investigación sustentada en los fundamentos teóricos de la microeconomía y de la economía del bienestar, así como de las restricciones que presenta el hecho de fijar precios basados en costos marginales. El modelo aplicado sugiere que el precio establecido por una empresa de servicios públicos, correspondiente al ámbito privado o público, puede incorporar a éste los efectos tanto de la distribución del ingreso como aquellos involucrados en la asignación de recursos, introduciendo en él ciertos juicios de valor explícitos.
\end{abstract}

Palabras clave: Eficiencia, economía de libre mercado, costo marginal, distribución del ingreso, utilidad marginal social.

\section{ABSTRACT}

The present work seeks to demonstrate that it is possible to obtain rates that incorporate the distribution of income and that simultaneously they area efficient. It is a work that is sustained in the theoretical foundations of the microeconomics and of the economy of welfare and of the restrictions that it presents to fix prices based on marginal costs. The model that is applied suggests that the price that a company of public services should establish, either private or public, is a price that takes into account, to a certain extent, effects on the distribution of Income, as well as the effects in the assignment of resources, introducing certain explicit judgements value.

Keywords: Efficiency, economy of market, maginal cost, distribution of income, social marginal utility.

\section{INTRODUCCIÓN}

Una economía de empresas privadas depende del mercado para poder asignar sus recursos. Los precios que se fijan en los mercados competitivos les proporcionan a los dueños o gerentes de empresas productoras la información para conocer los deseos de los consumidores, los cuales, a su vez, se encuentran con precios que son reflejo del costo de producción.

Se debe a los precios el hecho de que el mecanismo del mercado concilie los gustos de los consumidores con las condiciones técnicas de la producción y produzca una distribución de los recursos. Las técnicas de la producción dependen de los precios relativos de los factores, los cuales, a su vez, dependen de la facilidad y de la voluntad que los miembros de los hogares pongan en suministrarlos. La elección de las producciones la determinan los precios y los costos marginales, influidos por los gustos de los consumidores, por las posibilidades técnicas y por los precios de los factores.

Si se cumplen todas las suposiciones, incluyendo en ellas, muy especialmente, las suposiciones del máximo de ganancia y de la racionalidad de los consumidores, la colocación de los recursos que se producirán será lo que llamamos eficiente. Queremos decir con esto que la totalidad del aparato productivo de la economía funciona de tal modo que se logra el mayor beneficio para el consumidor, es decir, que éste logra las curvas más altas que le permiten alcanzar las posibilidades técnicas y en recursos de la economía [1].

Este concepto eficiencia se logra si los precios se ponen a nivel de los costos marginales.

\footnotetext{
${ }^{1}$ Universidad de Tarapacá, 18 de Septiembre 222, Arica-Chile, e-mail: dviera@uta.cl
} 
Las razones que hay que pensar que esta es una situación de máxima eficiencia son:

a) Todas las producciones se elaboran con el menor gasto de recurso, es decir, el mínimo de costo.

b) Los hogares suministran sus servicios en tal cantidad que las remuneraciones justamente compensan el servicio.

c) Al ser los costos marginales iguales a los precios, la proporción en que puede ser aumentada la producción de un artículo a expensas de otro es exactamente igual a la cantidad por la que los consumidores están dispuestos a cambiar, indiferentemente, un artículo por otro. Si las dos cantidades no fueran iguales, sería posible llevar a los consumidores a curvas más altas cambiando la composición de la producción.

La economía de libre mercado para lograr su máxima eficiencia requiere del cumplimiento de ciertos supuestos:

a) En primer lugar, los consumidores tienen que ser seres racionales; son personas que tienen preferencias y que son consecuentes con las mismas [2].

b) En segundo lugar, sus preferencias deben ser de tal naturaleza que su deseo de cambiar un artículo por otro, en respuesta a cambiar hechos en los precios, debe ir disminuyendo a medida que la sustitución se va realizando. Finalmente, las preferencias no deben tener nada que ver con las compras que hagan los demás, ya que, de lo contrario, el consumo que haga una familia puede ser causa de descontento en otra, descontento que no se refleja en los precios de las mercancías.

c) En lo referente a los productos de la economía, estos deben atenerse racionalmente al principio de lograr el máximo beneficio, y la producción se debe llevar adelante, de manera que las utilidades disminuyan. Si las utilidades aumentan, las empresas más fuertes de una industria podrían vender a precios más bajos que otros y destruir de esta manera la competencia. No debe haber ninguna interdependencia física entre los procesos de producción de empresas diferentes, ya que si la producción de una empresa influyera en la productividad de otras, aquella estaría creando o destruyendo beneficios para los consumidores del país, de una manera que no pertenece a sus cálculos sobre el obtener los mayores beneficios posibles.

d) El modelo también nos invita hacer suposiciones acerca de la estructura de los factores económicos y de los mercados de los productos. Los mercados deben ser perfectos. Con esto queremos decir que todos los que pertenecen a un mercado deben tener una información completa sobre sus precios y artículos, y que todos ellos, los de un lado y otro del mercado, deben tener tan poco poder que no puedan influir de ninguna manera en los precios que rigen el mercado.

e) La bondad del régimen de competencia presupone también la existencia de una apropiada distribución de ingresos. Si la distribución es mala, como juicio de valor, puede haber otras maneras de organizar el sector productivo que, quizá, no sean ideales desde el punto de vista de la eficiencia del sistema de producción y de la colocación de los recursos, pero que dan por resultado el lograr una mejor distribución de los ingresos. No se puede decir que esta alternativa sea peor, aunque su eficiencia sea menor, se podría crear una tercera situación, combinando la eficiencia del sistema de competencia con medidas de redistribución impuestas por el gobierno y que produjeran un reparto satisfactorio de una renta nacional. Esta combinación, entre el mejor arreglo productivo y una eficaz intervención del gobierno para garantizar una distribución justa de la renta, podría equivaler a la mejor situación económica posible.

f) El sistema de competencia, en su forma ideal, presupone también la existencia de una economía cuyos recursos son relativamente móviles. Si se dan diferencias en la productividad de un recurso destinado para varios usos, éste se debe destinar al uso en el cual es mayor la productividad. Cuando se trata de recursos materiales, la movilidad se debe al deseo de obtener un beneficio mayor.

g) En lo referido al recurso del trabajo, el caso es más complicado, porque el mercado del trabajo funciona en su parte informativa de una manera muy incompleta. Los trabajadores desconocen las oportunidades que hay en otras partes y dudan mucho antes de decidirse a un traslado.

Vamos a continuación a examinar las suposiciones que suelen ser más violadas. Señalaremos que esas violaciones justifican alguna forma de intervención gubernamental e indicaremos cómo se pueden adoptar en esos casos los principios de la economía del bienestar. Debemos subrayar el hecho de que no es necesario que la intervención gubernamental adopte las formas de una operación autoritaria y la convierta en apropiación, basta que el gobierno imponga ciertas normas y fije subsidios e impuestos para obtener resultados satisfactorios.

Suponemos existencia de competencia perfecta en todos los mercados, más aún en un mundo globalizado; sin embargo, en economías domésticas pequeñas con grandes concentraciones económicas y con empresas transnacionales es difícil que dicho supuesto se cumpla, más bien lo que realmente existen son mercados oligopólicos. 
En una situación como ésta es imposible fijar los precios igual al costo marginal, dado que éstos estarían por debajo de los costos medios (ver figura 1). Una forma de solucionar un problema de este tipo, en especial cuando se fijan tarifas de servicios públicos, es fijar los precios de manera discriminatoria, así los usuarios marginales paguen precios iguales a los costos marginales y otros tipos de usuarios paguen tarifas lo bastante altas como para obtener el cobro de todos los ingresos necesarios; o bien fijar precios que no cubran los ingresos necesarios pero que el Estado subvencione el déficit [3].

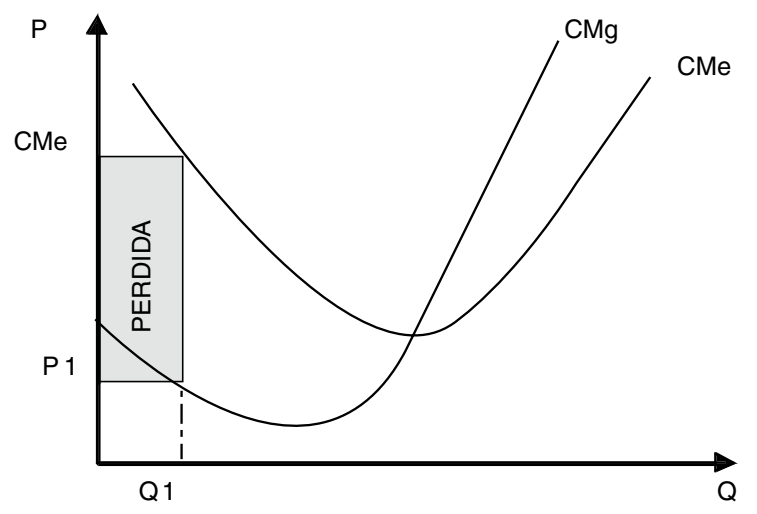

$\mathrm{CMg}=$ Costo Marginal

$\mathrm{CMe}=$ Costo Medio

Figura 1. Relación entre costos medios y costos marginales.

La pérdida la recuperan las empresas por intermedio de un cargo fijo.

Suponemos mutua independencia física: la dependencia mutua de diversos procesos productivos, fenómeno que a menudo recibe el nombre de economías externas o deseconomías de producción, también puede tener por efecto el hacer que los precios no sirvan para indicar los beneficios y costos marginales.

Si deseamos cumplir con la igualdad entre precios y costos marginales es necesario que la distribución del ingreso esté dada. Esta condición nos llevaría a la frontera de bienestar, pero al punto más favorable de ella; se requiere de impuestos globales y subvenciones para asegurar el movimiento deseado a lo largo la frontera. Muchas posiciones interiores a la frontera de bienestar representan un bienestar social más alto que muchas posiciones pertenecientes a la línea misma de la frontera. La distribución de los ingresos que se tiene del mecanismo de mercado no suele ser en manera alguna una distribución social. En nuestro sistema de propiedad privada tiene que hacerse alguna distribución con otros sistemas de proporcionalidad, el mecanismo del mercado tiene que producir una división diferente de la renta nacional. Es cuestión de juicios de valor el considerar qué tipo de distribución es buena y justa para un país.

Resumiendo, los principales problemas asociados con la determinación del precio igual al costo marginal son los siguientes:

1. El uso del precio igual al costo marginal implica una correspondencia directa entre el óptimo general y el resultado de la distribución del ingreso que no puede ser la óptima.

2. Condiciones que violan el principio del precio igual al costo marginal obligan algún tipo de intervención por parte del Estado, principalmente en las tarifas de los servicios públicos [4].

\section{MODELO}

\section{Algunas consideraciones del modelo}

El modelo se basará en los aspectos teóricos de la economía del bienestar. La aplicación del modelo es para las empresas de servicio público que producen con costos marginales por debajo de los costos medios.

El modelo plantea una serie de criterios generales que sirven como guía básica de la metodología utilizada. Tales criterios son:

a) El peso que tienen los servicios públicos dentro del gasto familiar no es pequeño; sin embargo, los efectos de cualquier política tarifaria en la distribución del ingreso siempre es pequeña, esto está significando que no se pretende mejorar la distribución del ingreso por medio de tarifas, pero sí fijar una tarifa que tenga en cuenta una mejora y que en ningún caso contribuya a empeorarla.

b) Con respecto a la distribución del ingreso, se construirán funciones de tipo paramétrico, que tendrán un factor de ponderación para los diferentes niveles de ingresos en los hogares.

c) Muy unido a la distribución del ingreso, introducimos el concepto de equidad, el cual puede verse desde dos puntos de vista:

- En forma vertical, que nos dice que quien tiene más o gana más debe pagar más. Esto constituye un juicio de valor reconocido socialmente como un hecho, justificando de esta forma las tarifas progresivas. 
- En forma horizontal, que nos dice que para ingresos iguales deben corresponder pagos iguales. Esto constituye el concepto de elasticidad horizontal.

- Por último se introduce la condición de que el precio debe ser por lo menos igual o mayor al costo marginal.

En lo que respecta a los principios del modelo podemos decir que éste está basado en el trabajo de Martin Feldstein [5] y el desarrollo de la determinación de precios óptimos para servicios públicos.

La más importante razón para seleccionar el modelo de Feldstein [3] es que posee una técnica de fabricar asignaciones con consideraciones distribucionales. Se trata de incluir efectos distribucionales en la evaluación de servicios públicos y podemos argumentar que estos efectos son importantes y pueden ser incorporados en el diseño de los precios y en la determinación de los costos.

El modelo prevé un mecanismo para solucionar este problema.

Otra razón es que el modelo está usando el excedente del consumidor como una aproximación del bienestar y además introduce el uso de un presupuesto limitado.

El modelo considera una regla para el precio que balancea la eficiencia y la equidad distribucional. Feldstein desarrolla una regla operacional óptima para el precio en términos de la demanda, parámetros distribucionales de fácil estimación y de un parámetro normativo básico de equidad distribucional.

\section{Determinación de un precio óptimo}

Si la distribución de los ingresos de los hogares está representada por la función de densidad $f(y)$, y si hay $N$ hogares en la población abastecida, el número de hogares con ingreso en un pequeño intervalo alrededor, yo es $N f\left(y_{0}\right) \cdot d y$. Se supone que la utilidad social marginal del ingreso para un hogar no es afectada por el precio marginal y carga fija anual. Esta aproximación parece justificada para cualquier aplicación práctica [6].

$\mathrm{P} \quad=$ Precio del servicio

$\mathrm{Q} \quad=$ Cantidad del servicio

$\mathrm{N} \quad=$ Total de hogares de la población abastecida

CT $=$ Costo total

$\mathrm{E}(p, y)=$ Excedente del consumidor

$f(y) \quad=$ Distribución de ingresos de los hogares
$N^{\prime}(y)=$ Utilidad marginal social de un peso para un hogar de ingreso $y$

A = Carga fija anual correspondiente a un individuo

$$
\mathrm{A}=[\mathrm{C}(\mathrm{Q})-\mathrm{p} \cdot \mathrm{Q}] \mathrm{N}^{-1}
$$

$\mathrm{V}=$ Valor neto del excedente de los consumidores de carácter agregado

Q = Podemos definirla como la cantidad total comprada cuando el precio marginal es

P, es decir:

$$
\mathrm{Q}=\mathrm{N} \int_{o}^{\infty} f(\mathrm{y}) \mathrm{Q}(\mathrm{p}, \mathrm{y}) \cdot \mathrm{dy}
$$

$\operatorname{MAX} \mathrm{V}=\mathrm{N} \int_{o}^{\infty} f(y) \mathrm{u}^{\prime}(y)\left[\mathrm{E}(p, y)-\mathrm{N}^{-1}[\mathrm{C}(\mathrm{Q})-\mathrm{p} \cdot \mathrm{Q}]\right] d y$

$$
\text { S.A. : } C(Q)-p \cdot Q \geq 0
$$

En el cálculo del precio óptimo esta restricción puede ser ignorada y $V$ maximizada directamente; si la solución viola la restricción, el precio puede ser reducido hasta que la restricción sea satisfecha.

Las condiciones de primer orden para un máximo de $V$ con respecto a $p$ son:

$$
\begin{aligned}
& \mathrm{N} \int_{o}^{\infty} f(y) \mathrm{u}^{\prime}(y)\left[\frac{\partial \mathrm{E}(p, y)}{\partial p}\right] d y= \\
& =\left[\left[\frac{\partial \mathrm{C}(\mathrm{Q})}{\partial \mathrm{Q}}-\mathrm{p}\right] \frac{\mathrm{dQ}}{\mathrm{dp}}-\mathrm{Q}\right] \int_{o}^{\infty} f(y) \mathrm{u}^{\prime}(y) d y
\end{aligned}
$$

Usando el resultado de Hicks [7] que $\frac{d \mathrm{E}(p, y)}{d p}=-\mathrm{Q}(p, y)$ y sustituyéndola por la expresión de Q, tenemos:

$$
\begin{aligned}
& \int_{o}^{\infty} f(y) \mathrm{u}^{\prime}(y) \mathrm{Q}(p, y) \cdot d y= \\
& =\left\{\left[p-\frac{\partial \mathrm{C}(\mathrm{Q})}{\partial \mathrm{Q} d p}\right] \frac{d \mathrm{Q}}{\mathrm{Q}} \cdot \underline{1}+1\right\} \int_{o}^{\infty} f(y) \mathrm{Q}(p, y) \cdot d y \int_{o}^{\infty} f(y) \mathrm{u}^{\prime}(y) d y
\end{aligned}
$$




$$
\begin{aligned}
& {\left[\frac{p-\frac{d(\mathrm{Q})}{d \mathrm{Q}}}{p}\right]\left[\frac{d \mathrm{Q}}{d p} \cdot \frac{p}{\mathrm{Q}}\right]=} \\
& =\frac{\int_{0}^{\infty} f(y) \mathrm{u}^{\prime}(y) \mathrm{Q}(p, y) \cdot d y-\int_{0}^{\infty} f(y) \mathrm{Q}(p, y) \cdot d y \int_{0}^{\infty} f(y) \mathrm{u}^{\prime}(y) d y}{\int_{0}^{\infty} f(y) \mathrm{Q}(p, y) \cdot d y \int_{0}^{\infty} f(y) \mathrm{u}^{\prime}(y) d y}
\end{aligned}
$$

La medida del lado izquierdo de la expresión anterior es el exceso del precio sobre el costo marginal multiplicada por la elasticidad precio de la demanda.

La elasticidad precio es negativa, el precio óptimo excede al costo marginal, sólo si el lado derecho es negativo.

El denominador del lado derecho es positivo. El numerador es la covarianza entre la cantidad consumida y la utilidad marginal social del ingreso a través de los hogares. Esta es negativa para bienes normales, es decir, cuando los ingresos aumentan disminuye la utilidad marginal del ingreso, la lógica de esto es: "Cargar más al costo marginal para familias de altos ingresos".

Para derivar una expresión explícita y operacional para el precio óptimo, la demanda de los hogares puede ser representada por la función:

$\mathrm{Q}(p, y)=\mathrm{a} \cdot y^{\alpha} \cdot p^{\beta}$

Con elasticidades ingreso y precio constante. El costo total puede ser expresado como:

$\mathrm{C}(\mathrm{Q})=\mathrm{F}+m \cdot \mathrm{Q}$

Sustituyendo en la expresión (5) se tiene:

$$
\left[\frac{p-m}{p}\right] \cdot \beta=\frac{\int_{o}^{\infty} f(y) \mathrm{u}^{\prime}(y) y^{\alpha} d y}{\int_{o}^{\infty} f(y) \mathrm{u}^{\prime}(y) d y f(y) \cdot y^{\alpha} d y}-1
$$

La razón de integrales de la expresión (6) es esencialmente la distribución paramétrica. Si llamamos a la distribución paramétrica por D, la ecuación (6) puede resolverse para el precio óptimo.

$$
\mathrm{P}=\frac{\beta}{\beta-(\mathrm{D}-1)} \cdot m
$$

El precio es proporcional al costo marginal para los bienes normales, (D-1) es negativo, B es negativa y por lo tanto el precio excede al costo marginal.

$$
\begin{gathered}
\frac{\mathrm{p}}{m}=\frac{\beta}{\beta-(\mathrm{D}-1)} \\
\frac{\partial\left[\frac{p}{m}\right]}{\partial \beta}=\frac{(\mathrm{D}-1)}{[\beta-(\mathrm{D}-1)]^{2}}>0
\end{gathered}
$$

Dado que

$$
\begin{aligned}
& (\mathrm{D}-1)<0 \\
& {[\beta-(\mathrm{D}-1)]^{2}>0}
\end{aligned}
$$

Tenemos que la expresión es mayor que cero. Por otro lado tenemos:

$$
\frac{{ }^{\partial}\left[\frac{p}{m}\right]}{\partial \mathrm{D}}=\frac{\beta}{[\beta-(\mathrm{D}-1)]^{2}}<0
$$

Expresando la elasticidad negativa que relaciona el nivel de ingreso con la utilidad social marginal como U'( $y$ ) de manera que:

$$
\mathrm{U}^{\prime}(y)=y^{-n}
$$

Siendo $n$ un término normativo paramétrico a determinar por decisiones políticas. De tomar por ejemplo un valor de cero, responderán igualmente los ingresos de todos los hogares independientemente de sus niveles de ingreso, ya que U'(y) vale 1.

La distribución paramétrica puede ahora ser escrita:

$$
\begin{aligned}
& \mathrm{D}=\frac{\int_{o}^{\infty} f(y) y^{\alpha} \cdot \mathrm{u}^{\prime}(y) \cdot d y}{\int_{o}^{\infty} f(y) y^{\alpha} \cdot d y \int_{o}^{\infty} f(y) y^{-n} \cdot d y} \\
& D=\frac{\int_{o}^{\infty} f(y) y^{\alpha-n} \cdot d y}{\int_{o}^{\infty} f(y) y^{\alpha} \cdot d y \int_{o}^{\infty} f(y) y^{-n} \cdot d y}
\end{aligned}
$$

Escribiendo $y^{\alpha-n}$ como exp $[\alpha-n] \ln y$, el numerador de $D$ es equivalente a la función generatriz de momentos del logaritmo del ingreso con parámetros $\alpha-n$. 
Similarmente, las integrales en el denominador son funciones generatrices de momentos con parámetros $\alpha \mathrm{y}-n$.

La distribución logarítmica normal provee una buena aproximación para la distribución de ingreso. Si Y tiene una distribución logarítmica normal.

$$
\int_{o}^{\infty} f(y) e^{a \log y} d y=\operatorname{Exp}\left[\alpha \cdot \bar{y}+\frac{1}{2} \alpha^{2} \sigma^{2} \bar{y}\right]
$$

Donde, $\bar{y}$ y $\sigma^{2}$ es la media y la varianza del logaritmo de $y$ respectivamente.

Evaluando los otros términos de la ecuación (12) y simplificando tenemos:

$$
\mathrm{D}=\operatorname{Exp}\left[\begin{array}{lll}
-\alpha \cdot n \sigma^{2} & - \\
&
\end{array}\right]
$$

Para una distribución logarítmica normal, la varianza del logaritmo puede ser expresada en términos del primer momento de la variable básica:

$$
\operatorname{Exp}\left(\sigma^{2} y\right)=1+\sigma^{2} y / u^{2}
$$

Si $V=\sigma^{2} y / u^{2}$, la distribución paramétrica $D$ puede escribirse como:

$$
\mathrm{D}=(1+\mathrm{V})^{-\alpha n}
$$

y el precio óptimo como:

$$
P=\frac{\beta}{\left[\beta+1-(1+V)^{-a n}\right]} \times m
$$

La ecuación (17) provee una fórmula operacional para calcular el exceso del precio sobre el costo marginal, como una función del parámetro normativo n; la función de demanda tiene los parámetros y el coeficiente de variación de la distribución del ingreso. Es claro por la ecuación que el precio se incrementa por la subida de la varianza relativa al ingreso, la elasticidad ingreso de la demanda y la elasticidad absoluta de la función de utilidad marginal.

Para que el precio no sea negativo se requiere que:

$$
(\mathrm{V}+1)^{-\alpha n}>1+\beta
$$

La expresión (18) nos provee una fórmula para determinar el precio como una modificación del costo marginal que difiere para cada nivel de ingreso familiar.

\section{Aplicación del modelo al agua potable en Arica}

Esta última parte muestra el funcionamiento del modelo elaborado por M. S. Feldstein, y que permite analizar los conceptos de eficiencia y equidad. En este análisis del precio por los servicios de agua potable se determinan una serie de precios correspondientes a diferentes valores de la elasticidad de la utilidad marginal social del ingreso, de este modelo se pretende mostrar la sensibilidad del precio óptimo ante cambios del parámetro normativo y así identificar explícitamente el rol que juega la equidad en las decisiones del precio óptimo.

El modelo está pensado para situaciones donde la empresa presenta costos marginales por debajo de los costos promedios. Existen razones para pensar que la empresa tenga esta característica en primer lugar, las inversiones realizadas soy muy superiores a la demanda, en segundo lugar, al ser rígida la capacidad con incrementos en los costos de carácter proporcional especialmente en lo que respecta a productos químicos y materiales y fijo en lo referente a los gastos de mantención; es fácil de suponer que los costos medios son superiores a los costos marginales. Aquí se da el caso de la indivisibilidad de uno de los factores de la producción, que se relaciona directamente con los rendimientos y los costos totales.

Dadas las limitaciones técnicas de una empresa de agua potable es posible aumentar algún factor, pero es imposible seguir aumentando la producción; esta situación unida a que muchos de los gastos, como son de mantenimiento y los de sueldos, están determinados para la capacidad máxima, que no es en ningún momento igual a la oferta, dado que permanece capacidad ociosa, para posibles demandas pico, crecimiento poblacional o pérdidas que se presentan.

\section{Determinación de los parámetros del modelo}

En la obtención de las elasticidades precio e ingreso de la demanda se utilizó como especificación una función de tipo exponencial, usando una Cross-Section por quintiles de ingreso para los años 1998, 1999 y 2000.

La especificación utilizada fue la siguiente:

$$
\begin{aligned}
& \mathrm{L} n \mathrm{C}=\mathrm{LnA}+\alpha \operatorname{Lny}+\beta \operatorname{Ln} p+\mathrm{L} n \mathrm{E} \\
& \mathrm{L} n \mathrm{C}=-5,565+0,898 \mathrm{Ly}-0,446 \operatorname{Ln} p+\mathrm{L} n \mathrm{E}
\end{aligned}
$$$$
(1,591) \quad(0,018) \quad(0,243)
$$ 
Donde:

$$
\begin{aligned}
C= & \text { Es el consumo expresado en términos de } \\
& \mathrm{M}^{3} / \text { mes } \\
p= & \text { Precio por } \mathrm{M}^{3} \\
y= & \$ / \text { mes con base año } 2002 \\
E= & \text { Término de error } \\
\alpha= & \text { Elasticidad ingreso de la demanda } \\
\beta= & \text { Elasticidad precio de la demanda }
\end{aligned}
$$

Los resultados estadísticos se pueden interpretar de la forma siguiente:

En primer lugar, el signo de los coeficientes está de acuerdo a la teoría económica, es decir, la elasticidad precio de la demanda es menor que cero y la elasticidad precio de la demanda mayor que cero.

Para analizar si existe evidencia estadística de que por lo menos un parámetro no es igual a cero a un cierto nivel de significancia, se utilizó la prueba $\mathrm{F}$. Con respecto a los coeficientes $\alpha$ y $\beta$ se utilizó la prueba $t$, resultando $\beta$ significativa a un $5 \%$ y el coeficiente $\alpha$ significativa a un $9,1 \%$. Sin embargo, como el estudio que se está realizando no es de carácter estructural más bien de índole normativa, consideraremos la significancia de $\alpha$ como válida.

En base a la información de los ingresos por quintiles para el período año 1996 al 2000, se determinó el coeficiente de variación del ingreso

$$
V=\sigma^{2} y / \bar{y}=0.64
$$

Las tarifas a simular son función de las elasticidades precio de la demanda, elasticidad ingreso, del coeficiente de la variación del ingreso, cargo fijo por cliente, cargo variable por producción agua en período no punta y de un concepto normativo.

$\mathrm{CF}=$ cargo fijo por cliente

$\mathcal{M}=$ Cargo variable por producción $\quad \$ 237,37 / \mathrm{M}^{3}$

Al precio no se le ha considerado como función del costo adicional producido por una demanda pico; su tratamiento no dificulta el análisis dado que al costo adicional se le agrega el costo marginal.

\section{CONCLUSIONES}

Con el fin de evitar una regresividad en las tarifas el modelo introduce la distribución del ingreso con el propósito de fijar tarifas discriminadas por hogares; esto se logra dándole diferentes ponderaciones del peso gastado y que se expresa en la elasticidad negativa que relaciona el nivel de ingreso con la utilidad social marginal [u'(y)], a medida que aumentamos el término normativo $(n)$ por decisiones políticas, se tiene que el cargo variable por $\mathrm{M}^{3}$ aumenta y la parte fija decrece en relación a la facturación total, lo que nos soluciona los problemas de regresividad de una tarifa en dos partes sin consideraciones distribucionales (Ver cuadro 1).

Estos conceptos tienen una fuerte repercusión en lo que respecta a la función de bienestar a ser maximizados, expresados a través del excedente del consumidor.

El modelo de Feldstein considera al excedente del consumidor agregado y ponderado como una buena aproximación del bienestar de la comunidad. Con el fin de cuantificarlo se han utilizado las siguientes expresiones:

$$
\begin{gathered}
\text { Cambio en el excedente }\left(E_{i}\right)=\mathrm{Q}_{0} \Delta P+\frac{\mathrm{P} \times \mathrm{Q}}{2} \\
w\left(y_{i}\right)=\frac{I}{Y_{i}^{n}}
\end{gathered}
$$

Donde $i$ está representando a un grupo de hogares de un ingreso dado y de un quintil determinado.

De los cuadros 2, 3 y 4 se observa que existe un aumento del bienestar de la comunidad que no es en ningún momento compensado con las pérdidas de bienestar de los grupos de más altos ingresos, a pesar de haber considerado diferentes valores para el término normativo $(n)$. Esto afirma una vez más la necesidad de considerar aspectos distribucionales en la fijación de tarifas en dos partes de modo de solucionar el problema de la equidad y de la eficiencia cuando se tienen costos medios superiores a los costos marginales. Fijar precios eficientes basados en costos marginales resulta restrictivo dado que los supuestos en que se sustenta no se cumplirán en la práctica; por lo tanto, lo que pretendemos sugerir con este trabajo es que el precio que debería establecer una empresa de servicios públicos es un precio que atienda hasta cierto punto sus efectos sobre la distribución del ingreso, como también los efectos en la asignación de recursos, introduciendo ciertos juicios de valor explícitos.

La asignación de recursos es eficiente desde una concepción económica social, dado que estas empresas pueden alcanzar presupuestos eficientes sin empeorar la distribución del ingreso, dado que la regresividad de la parte fija estaría siendo eliminada. 


\section{REFERENCIAS}

[1] Kennet J. Arrow. "Una dificultad en el concepto de Bienestar Social". Ensayos sobre Economía del Bienestar. FCE. México. 1974.

[2] J.R. Hicks. "A revision of demand Theory". Oxford the Clarendon Press. 1956.

[3] M. Boitem. "Peak-load Pricing”. Marginal cost pricing in practice. J.R. Nelson (ed.). Prentice Hall. Englewood Cliffs. NJ, pp. 59-90. 1964.

[4] J.R. Nelson. "Marginal Cost Pricing in Practice". Prentice Hall. Englewood Cliffs. NJ. 1964.
[5] Martin S. Feldstein. "Equity and Efficiency in Public Sector Pricing: The optimal Two-part-tariff". Quarterly Journal of Economic. LXXXVI, pp. 175187. May 1972.

[6] J.R. Hicks. "La rehabilitación del excedente de los consumidores. Ensayo sobre economía del bienestar". F.C.E. México, pp. 405-417. 1974.

[7] J.R. Hicks. "Value and Capital". 2nd Ed. London: Oxford at the Clarendon Press. 1946.

\section{CUADRO 1 \\ VARIACIÓN DE LA PARTE VARIABLE CARGO FIJO Y TARIFA}

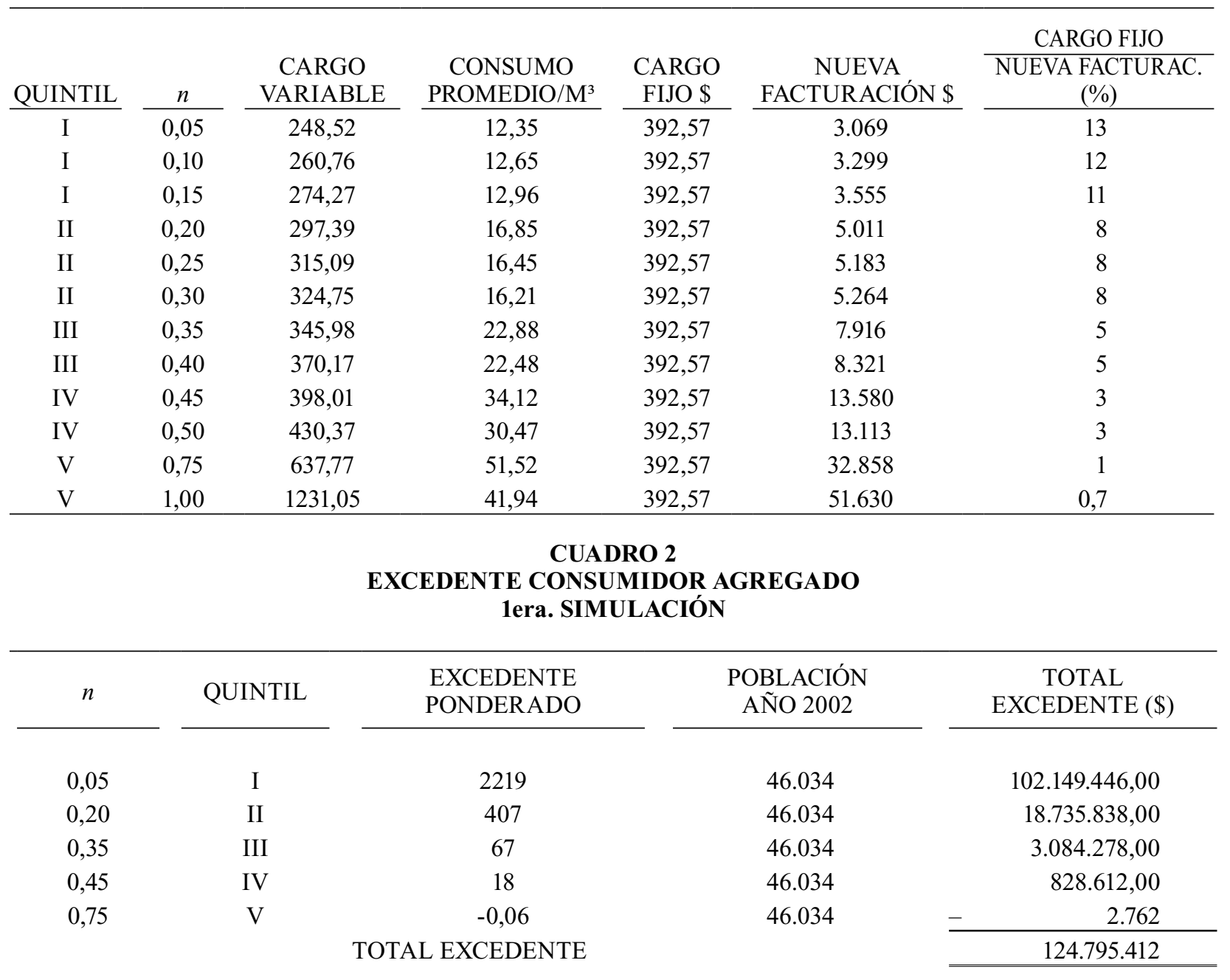




\section{CUADRO 3 \\ EXCEDENTE CONSUMIDOR AGREGADO \\ 2da. SIMULACIÓN}

\begin{tabular}{|c|c|c|c|c|}
\hline$n$ & QUINTIL & $\begin{array}{l}\text { EXCEDENTE } \\
\text { PONDERADO }\end{array}$ & $\begin{array}{l}\text { POBLACIÓN } \\
\text { AÑO } 2002\end{array}$ & $\begin{array}{c}\text { TOTAL } \\
\text { EXCEDENTE }(\$)\end{array}$ \\
\hline 0,10 & I & 1.405 & 46.034 & 64.677 .770 \\
\hline 0,25 & II & 253 & 46.034 & 11.646 .602 \\
\hline 0,40 & III & 40 & 46.034 & 1.841 .360 \\
\hline 0,50 & IV & 10 & 46.034 & 460.340 \\
\hline \multirow[t]{2}{*}{1,00} & $\mathrm{~V}$ & $-0,03$ & 46.034 & 1.381 \\
\hline & & AL EXCEDENTE & & 78.624 .691 \\
\hline
\end{tabular}

\section{CUADRO 4 \\ EXCEDENTE CONSUMIDOR AGREGADO 3ra. SIMULACIÓN}

\begin{tabular}{|c|c|c|c|c|}
\hline$n$ & QUINTIL & $\begin{array}{l}\text { EXCEDENTE } \\
\text { PONDERADO }\end{array}$ & $\begin{array}{l}\text { POBLACIÓN } \\
\text { AÑO } 2002\end{array}$ & $\begin{array}{c}\text { TOTAL } \\
\text { EXCEDENTE }(\$)\end{array}$ \\
\hline 0,15 & I & 795 & 46.034 & 36.597 .030 \\
\hline 0,30 & II & 137 & 46.034 & 6.306 .658 \\
\hline 0,40 & III & 43 & 46.034 & 1.979 .462 \\
\hline 0,50 & IV & 11 & 46.034 & 506.374 \\
\hline 1,00 & $\mathrm{~V}$ & $-0,002$ & 46.034 & 92 \\
\hline & & AL EXCEDENTE & & 45.389 .432 \\
\hline
\end{tabular}

\section{CUADRO 5 \\ EXCEDENTE CONSUMIDOR AGREGADO 4ta. SIMULACIÓN}

\begin{tabular}{|c|c|c|c|c|}
\hline$n$ & QUINTIL & $\begin{array}{l}\text { EXCEDENTE } \\
\text { PONDERADO }\end{array}$ & $\begin{array}{l}\text { POBLACIÓN } \\
\text { AÑO } 2002\end{array}$ & $\begin{array}{c}\text { TOTAL } \\
\text { EXCEDENTE (\$) }\end{array}$ \\
\hline 0,15 & I & 682 & 46.034 & 31.395 .188 \\
\hline 0,30 & II & 140 & 46.034 & 6.444 .760 \\
\hline 0,40 & III & 61 & 46.034 & 2.808 .074 \\
\hline 0,50 & IV & 1 & 46.034 & 46.034 \\
\hline 1,00 & $\mathrm{~V}$ & $-0,003$ & 46.034 & 138 \\
\hline & & AL EXCEDENTE & & 40.693 .918 \\
\hline
\end{tabular}


CUADRO 6

DETERMINACIÓN DEL EXCEDENTE DEL CONSUMIDOR

\begin{tabular}{|c|c|c|c|c|c|c|c|c|c|c|c|c|}
\hline AÑO & QUINTIL & (i) & $\begin{array}{c}\mathrm{N}^{\circ} \text { HOGARES } \\
\text { MUESTREADOS }\end{array}$ & $\begin{array}{l}\text { INGRESO } \\
\text { PROMEDIO }\end{array}$ & $\mathrm{n}$ & $\begin{array}{c}\text { TARIFA } \\
\left.\text { ACTUAL /(M }{ }^{3}\right)\end{array}$ & $\begin{array}{c}\text { CONSUMO } \\
\text { ACTUAL/(M³) }\end{array}$ & $\begin{array}{c}\text { NUEVA } \\
\left.\text { TARIFA/(M }{ }^{3}\right)\end{array}$ & $\begin{array}{c}\text { NUEVO } \\
\left.\text { CONSUMO/( } \mathrm{M}^{3}\right)\end{array}$ & $W(i)$ & $(E x)$ & $\begin{array}{l}\text { EXCEDENTE } \\
\text { PONDERADO }\end{array}$ \\
\hline 1998 & I & 1 & 26 & 129.362 & 0,05 & 614 & 8,6 & 249 & 12,30 & 0,56 & 3.996 & 2.219 \\
\hline 1999 & I & 2 & 26 & 136.012 & 0,10 & 677 & 8,4 & 261 & 13,62 & 0,31 & 4.580 & 1.405 \\
\hline 2000 & I & 3 & 26 & 142.662 & 0,15 & 695 & 8,5 & 274 & 13,91 & 0,17 & 4.718 & 795 \\
\hline 1998 & II & 4 & 63 & 193.909 & 0,20 & 614 & 11,6 & 297 & 17,68 & 0,09 & 4.641 & 407 \\
\hline 1999 & II & 5 & 63 & 193.668 & 0,25 & 677 & 12,1 & 315 & 17,20 & 0,05 & 5.304 & 253 \\
\hline 2000 & II & 6 & 63 & 193.467 & 0,30 & 695 & 11,6 & 325 & 16,95 & 0,03 & 5.282 & 137 \\
\hline 1998 & III & 7 & 44 & 287.758 & 0,35 & 614 & 17,1 & 346 & 23,54 & 0,01 & 5.446 & 67 \\
\hline 1999 & III & 8 & 44 & 289.479 & 0,40 & 677 & 17,3 & 371 & 22,94 & 0,01 & 6.157 & 40 \\
\hline 2000 & III & 9 & 44 & 291.200 & 0,40 & 695 & 17,9 & 371 & 23,07 & 0,01 & 6.636 & 43 \\
\hline 1998 & IV & 10 & 37 & 475.001 & 0,45 & 614 & 26,7 & 398 & 34,69 & 0,003 & 6.629 & 18 \\
\hline 1999 & IV & 11 & 37 & 455.005 & 0,50 & 677 & 24,7 & 430 & 32,24 & 0,001 & 7.032 & 10 \\
\hline 2000 & IV & 12 & 37 & 435.009 & 0,50 & 695 & 24,6 & 430 & 30,97 & 0,000 & 7.363 & 11 \\
\hline 1998 & $\mathrm{~V}$ & 13 & 34 & 437.759 & 0,75 & 614 & 54,6 & 638 & 26,12 & 0,000 & -968 & $-0,06$ \\
\hline 1999 & $\mathrm{~V}$ & 14 & 34 & 1.031 .518 & 1,00 & 677 & 53,4 & 1.231 & 42,06 & 0,000 & -26.443 & $-0,03$ \\
\hline 2000 & V & 15 & 34 & 1.125 .278 & 1,00 & 695 & 50,8 & 1.231 & 45,48 & 0,000 & -25.803 & $-0,002$ \\
\hline
\end{tabular}

